\title{
La función atenuante de la partícula discursiva ¿eh? en el lenguaje adolescente de Madrid
}

\author{
Annette Myre Jørgensen (Escuela Superior de Østfold, Noruega)
}

\begin{abstract}
The discourse particle $e h$ has a high frequency in the Madrid teenage talk. The different functions this short particle displays in the interaction, as for instance: calling upon the hearer's attention, demanding approval or asking for a repetition of what is said, or hedging is worth some attention. One of the many functions attributed to this particle is the hedging one, especially when there are utterances containing speech acts conveying orders, critics and recriminations, which could imply a face threat. This paper will therefore describe the hedging function of the final interrogative $; e h$ ? in the oral speech among Madrid teenagers. Hedging is a pragmatic strategy by which the speaker minimizes the effects of the utterance. It can have impact on different elements in the communicative process: the message, the speaker, the hearer and the relation between both. Although teenagers are not known for their tendency to soften the content and effect of their sayings, there are several utterances among the Madrid teenagers that present a certain hedging through the particle $¿ e h$ ? in a final position.

The examples used in this study are extracted from the Corpus Oral de Lenguaje Aolescente, COLA-corpus (http://www.colam.org/), which contains spontaneous, informal teenage (13 to 19 years) talk from Madrid. There are more than 5000 concordances with $i e h$ ? displaying a wide array of functions in different positions in the utterance. The cases that have $\measuredangle e h$ ? in a final position and a hedging function have been selected for the analysis of this paper.
\end{abstract}

Keywords: corpus, teenage-talk, hedging, discourse markers, face-threats

\section{Introducción}

La partícula discursiva ¿eh? está presente de modo notorio en el lenguaje adolescente de Madrid. Tiene una amplia gama de funciones discursivas que parten de la principal: mantener el contacto con el interlocutor (Santos Ríos 2003). La presencia de ¿eh? permite al hablante pedir la aprobación del oyente, solicitar repetición de lo dicho, reforzar una orden y mitigar una afirmación negativa o una reprensión. Uno de los valores de ¿eh? que llama la atención es su efecto atenuante en actos de habla como recriminaciones, críticas, rechazos, advertencias y órdenes, que pudieran suponer una amenaza a la imagen. 


\subsection{Objetivos del trabajo}

Sorprende que una palabra tan reducida y versátil en cuanto a posición y entonación pueda desplegar tan rico registro de funciones. El valor atenuante de la partícula ¿eh? no ha hecho derramar mucha tinta, lo que invita a abordar este aspecto desde una perspectiva pragmático-discursiva. Este trabajo se propone dar cuenta de la función discursiva atenuante de la partícula ¿eh? en la interacción oral, informal, adolescente de Madrid. Dado que no se ha analizado la función atenuante de ¿eh? anteriormente en el lenguaje adolescente de Madrid, esta meta no está excenta de retos, ya que los jóvenes adolescentes no destacan por su tendencia a las afirmaciones suavizadas (Briz 2003, Jørgensen 2009, Cestero 2012). No obstante, existen intervenciones atenuadas mediante la partícula ¿eh? realizadas por adolescentes madrileños, por ejemplo, la siguiente crítica:

Manu: no hue\% no hueles el peligro ¿eh? (MALCE2)

o la advertencia:

Mar: ahora no puedes ir a comprarlo ¿eh? (MABPE2)

\subsection{Delimitación del tema}

El corpus COLAm reúne 2311 enunciados con el apéndice interrogativo ¿eh? en posición final de la unidad discursiva que mantiene la comunión fática y controla que lo que se dice está siendo seguido por el oyente, intensifica una recriminación o solicita ratificación de lo dicho. La partícula eh no-interrogativa también es usada en la interacción juvenil para retener el turno mientras se piensa lo que se va a decir, gestionar los turnos o llamar la atención (Santos Ríos 2002: 253). Mencionadas funciones no se verán en este trabajo, que se ciñe a los 111 casos de la partícula interrogativa ¿eh? con valor atenuante, con entonación ascendente o descendiente al final del enunciado.

\subsection{Estructura del trabajo}

Este trabajo tiene cuatro partes; en la primera se presenta el objetivo y la delimitación del tema, así como los motivos y la estructura del trabajo. En la segunda parte se explica el marco metodológico y el teórico. El tercer apartado se dedica al análisis de la atenuación con ¿eh? Las conclusiones se recogen en el último y cuarto epígrafe.

\section{Marco metodológico y teórico}

Zimmermann afirma que el estudio del lenguaje adolescente se tiene que basar en intervenciones auténticas: "La base de partida para su estudio ha de ser el acto comunicativo, la conversación de o entre jóvenes: no pueden tomarse los elementos por separado o de manera aislada [...]" (2002: 141). Albelda, por su parte, (2010b) señala que el problema de la identificación de los enunciados atenuados estriba en que, a pesar de que sus formas y funciones se encuentran, con más o menos suerte, establecidas, la atenuación es una categoría pragmática que solo es posible identificarla en su contexto real. 


\subsection{El corpus COLAm}

Briz y Albelda apuntan a la conveniencia de ver las funciones atenuantes en diferentes corpus de conversaciones coloquiales e informales (2013). El corpus COLAm, Corpus Oral de Lenguaje Adolescente de Madrid, presenta enunciados reales transcritos, unidos al sonido, lo que, sin duda, facilita el análisis de la atenuación, aunque no sea siempre fácil para el investigador reconocer la atenuación, a pesar de trabajar con un corpus (Albelda 2010:1). Las conversaciones se han mantenido sin presencia de adultos, lo que asegura la espontaneidad de las conversaciones (Jørgensen y Eguía Padilla 2015, Jørgensen 2013). Los casos de ¿eh? con la función pragmático-discursiva de atenuante, en los que se basa este estudio, pertenecen a este corpus, que tiene alrededor de 500.000 palabras en total. Las transcripciones tienen un mínimo de codificación ${ }^{1}$.

Este trabajo se inserta dentro de un método empírico inductivo, cualitativo y cuantitativo, basado en la descripción de las funciones pragmático-discursivas de la partícula ¿eh? así como el cómputo de la frecuencia de estas en el corpus COLAm.

Los turnos en las conversaciones adolescentes son breves y los intercambios rápidos, con frecuentes cambios de temas. Los enunciados constituyen generalmente actos de habla en intervenciones iniciativas o reactivas que forman los turnos (Val.Es.Co, 2014).

\subsection{Marco teórico}

El efecto de las funcionesde la partícula discursiva ¿eh? en la interacción coloquial ha sido observado con acierto en el Diccionario de Partículas de Santos Ríos (2003), en los trabajos de Montañez Mesas (2014, 2015), y en el Diccionario de Partículas Discursivas del español (Briz y Pons. 2008). En estos tres trabajos sale a relucir el valor de atenuación. Los principios teóricos y metodológicos que proponen Albelda (2010a) y Briz y Albelda (2013) Cestero, Albelda y Briz (2015) fundamentan el análisis del valor atenuante del ¿eh? en las interacciones juveniles en este trabajo.

\subsection{Las partículas discursivas o apendices interrogativos}

Desde que Ortega Olivares abordara el tema de los apéndices interrogativos (1985, 1986) se ha tratado el tema en muchos trabajos sobre el español oral (García Vizcaíno 2005, Cestero 2003, Gille 2006, Montañez Mesas 2007, Montañez Mesas 2008, Blas Arroyo 1995, Vigara Tauste 1990) tanto en el lenguaje coloquial, como en el académico (Cestero 2003). Para un estado de la cuestión, remito a las exposiciones sobre eh en las tesis de Edelso Natalías (2006) y Montañez Mesas (2015).

Stenström (2005b), Montañez Mesas (2007) y Blas Arroyo (1995) afirman que el fin del uso de este tipo de partícula es mantener la relación interpersonal o la comunión fática. Santos Ríos (2003) apunta a las funciones de ¿eh? como un recurso lingüístico para llamar la atención al interlocutor, mantener la comunión fática y controlar que lo que se dice está siendo seguido por el oyente. Blas Arroyo (1995) expone un elenco de las diferentes funciones de eh, partiendo del hecho de que ¿eh? es clave para la interacción.

\footnotetext{
${ }^{1}$ El corpus COLAm está transcrito ortográficamente, dispuesto para escuchar el archivo de sonido a la vez que se leen las transcripciones. Las señales de transcripción son: [ ] Solapamiento. XXX - Habla poco clara, \% Palabra interrumpida. / Entonación ascendente de pregunta. I. Entonación ascendente de pregunta/. Pausa de un segundo .. Pausa de dos segundos ... Pausa de tres segundos.
} 
La proliferación de las partículas controladoras de contacto en el lenguaje adolescente no debe sorprender. Queda establecido en numerosos trabajos que el fin primordial de la interacción adolescente es social (Briz 2003, Blas Arroyo 2005, Montañez Mesas 2007), por un lado y, por otro, que hay (ab)uso de los marcadores del discurso entre los jóvenes (Jørgensen y Martínez 2007, Stenström 2005, Rodríguez, 2001).

Hay acuerdo entre los especialistas que se han acercado a esta partícula de un modo más detenido (Santos Ríos 2003, Blas Arroyo 1995, Montañez Mesas 2007) en que la partícula discursiva $₫ e h ?$ no forma parte del terreno sintáctio.

\subsection{La teoría de la atenuación}

La atenuación ha sido objeto de una larga trayectoria de investigación en la pragmática, en general, y en el español coloquial, en especial (Briz y Pons 2008, Briz 2010, Albelda 2005, Albelda 2010c, Albelda y Briz 2013, Albelda Marco 2008, Cestero, Albelda, y Briz 2015). Según Briz y Albelda, la atenuación es "un mecanismo retórico para convencer, lograr un beneficio, persuadir y, a la vez, para cuidar las relaciones interpersonales y sociales o evitar que estas sufran algún tipo de menoscabo" (2013: 293).

Siendo una estrategia pragmática al servicio de la comunicación mediante la que se pretende minimizar el efecto de lo dicho o lo hecho, la presencia de atenuadores puede afectar a diversos elementos del proceso comunicativo: al mensaje, al hablante, al oyente o a la relación entre ambos. La atenuación incide en lo dicho, afectando a la dimensión semánticopragmática y en el decir, en el modus, en la dimensión más propiamente pragmática (Briz 1998: 148-150; 2007: 12-13).

\subsection{La atenuación en la interacción adolescente}

La interacción adolescente prototípica del corpus COLAm es la informal mantenida entre amigos. La cortesía que caracteriza a este tipo de interacción es la de los miembros que pertecen al mismo grupo, tal y como afirman Brown y Levinson (1987) y, posteriormente, Carter y Mc Carthy (2006). El fin de la interacción es social (Herrero 2002, Zimmermann 2002, Briz 2003, Jørgensen 2011) o, con otras palabras, mantener la comunión fática (Malinowski 1999 [1926], Vigara Tauste 1990). Briz y Albelda insisten en el fin social de la conversación:

En la conversación, como en la mayoría de los discursos, la función predominante es la que hemos convenido en llamar función fático-expositiva, vinculada al fin interpersonal que predomina en toda conversación coloquial (aquella conversación que busca la comunión con el otro, el aumento de las relaciones interpersonales y sociales) (2013: 310).

Los actos de habla que pudieran conllevar amenazas a la imagen y una consecuente ruptura de la comunión fática en otros contextos explican, en nuestro contexto particular, la escasa frecuencia de atenuantes, dada la relación existente entre los miembros y su tipo de interacción. «A mayor coloquialidad, en principio, menor actividad atenuadora» (Briz y Albelda: 2013: 294). 


\subsection{El valor de atenuación de la partícula discursiva ¿eh?}

Hay una clara dificultad en cuanto a la delimitación de la función discursiva de atenuante: los marcadores discursivos, en general, y la partícula ¿eh?, en particular, son polifuncionales. Por ello, son convenientes los criterios propuestos por Albelda (2008, 2010) y Briz y Albelda (2013), que sugieren detenerse en los siguientes rasgos situacionales:

Rasgos situacionales

Marco físico donde se realiza la entrevista o conversación
(-) Formal

Familiar/ cotidiano

Igualdad funcional y/o social

Relación vivencial de proximidad: amigos, parientes, colegas, conocidos

\section{(+) Formal}

Transnacional

\section{Relación de poder/ jerarquía entre los} interlocutores

Grado de proximidad, conoci-miento común compartido entre los interlocutores
Acercamiento
Desigualdad funcional y/o social

Relación vivencial de no proximidad (desconocimiento)

Origen geográfico y cultural del hablante. Culturas de:

Tabla I: Rasgos situacionales ( \pm Formal) de la atenuación

Para la correcta identificación de la atenuación, resulta necesario prestar atención no solo a los rasgos situacionales si no a la relación que hay entre los participantes de la conversación. Las conversaciones de los adolescentes del corpus COLAm tienen lugar en casa de alguno del grupo, por la calle, en cafeterías, en el recreo, en el campo, en parques, etc. Hablan con sus compañeros de clase de la misma edad y estrato social (Jørgensen y Eguía 2015), por lo que hay un elevado grado de relación vivencial de proximidad y de mundo compartido entre los hablantes. Varios estudios (Jørgensen y Drange 2012) indican que hay una cultura de acercamiento entre ellos, por ser españoles y por ser amigos. Es sabido que los adolescentes tienen una dependencia marcada de sus amigos y grupos de amigos (Gina Tomé et al. 2012, Brown y Larson 2009, Eckert 2003), lo que lleva a concluir que el lenguaje adolescente del corpus COLAm reúne el rasgo situacional informal: (-) Formal.

En segundo lugar, cabe atender al tipo de acto de habla y a la fuerza ilocutiva que se desprende de este, para lo que hay que acudir "a las plantillas de mecanismos y formas de atenuación" (Albelda 2010b: 11). En tercer lugar, hay que mirar la implicación de las imágenes de los hablantes porque «los tipos de atenuación que resultan más fácilmente identificables son, en su mayoría, aquellos en que se implican las relaciones de imágenes personales, pues es esta la principal causa de empleo de atenuación" (Albelda 2010b:12). El cuarto aspecto que se ha considerado son los actos asertivos atenuados, en cuyo análisis conviene diferenciar si son opiniones o aserciones de hechos factuales, discerniendo «si la intención del hablante realmente contiene duda o si atenúa para comprometerse menos" (Albelda 2010b:12). En quinto lugar y último lugar, hay actos de habla asertivos atenuados que protegen la imagen del oyente o la imagen del hablante. En Briz y Albelda (2013) se añade otro aspecto para la identificación: "nos referimos al momento o a los momentos puntuales de habla en una interacción que afectan, favorecen o llegan a determinar los usos y estrategias lingüísticas". La noción de contexto interaccional concreto dinamiza, según Briz y Albelda, el concepto más general de situación de comunicación: "el miembro del discurso 
afectado por la atenuación, el miembro del discurso causante o desencadenante, la expresión atenuante concreta y, en su caso, el efecto que produce" (2013: 12).

Se puede, asimismo, analizar el enunciado dejando el espacio de la partícula ¿eh? vacío o hacer una sustitución por otro marcador discursivo atenuante (Blas Arroyo, 1995). En el caso del corpus COLAm ha sido importante escuchar el archivo de sonido de cada conversación y contexto de aparición de ¿eh? (Briz y Albelda 2013), que, según Hidalgo, “en los usos atenuadores (e incluso en los no (des)corteses) hay un claro predominio del contorno entonativo ascendente con o sin reajuste" (2016: 99).

\section{Análisis de los valores de ¿eh?}

En este trabajo se observan las frecuencias de la función atenuante de ¿eh? en posición final de enunciado entre los adolescentes del corpus COLAm. Es el apéndice interrogativo más frecuentemente usado en las situaciones descritas.

\subsection{Los valores de ¿eh? en los enunciados del corpus COLAm}

En total, se han registrado 2311 casos de ¿eh? en posición final con las siguientes funciones: solicitar ratificación de lo dicho; reforzar la alianza; reforzar el argumento del hablante; mantener la atención del interlocutor; intensificador ante exhortaciones, órdenes y advertencias o atenuante ante órdenes, advertencias y críticas:

\section{Función $\quad \mathbf{N}^{0}$ casos $\%$}

1. El hablante solicita al oyente ratificar lo que le dice/pide

Reforzador de la alianza.

Mantenedor la atención del interlocutor.

$1031 \quad 44,6$

Reforzador de los argumentos del hablante.

2. Intensificador ante exhortaciones, órdenes, advertencias.

$1169 \quad 50,6$

3. Atenuante ante críticas, órdenes, advertencias.

1114,8

TOTAL

$2311 \quad 100$

Tabla II: Las tres principales funciones de ¿eh? en posición final

La tabla II indica que el número de actos de habla atenuados por ¿eh? en el corpus COLAm es poco bajo, ya que de la totalidad de 2311 casos de ¿eh? solo 111 presentan un valor de atenuación, lo que supone una proporción de 4,8\% del total. Esto no debe sorprender debido no solo a los rasgos propios del habla adolescente, que tiende más a la hipérbole y a la exageración que a la atenuación (Briz 2003, Jørgensen 2009), sino también por el tipo de interacción. En los enunciados de los adolescentes la función atenuante de ¿eh? está frecuentemente acompañada de otras funciones (Briz y Albelda 2013, Jørgensen 2007). La proporción relativa entre las funciones de ¿eh? se representa en la tabla III: 


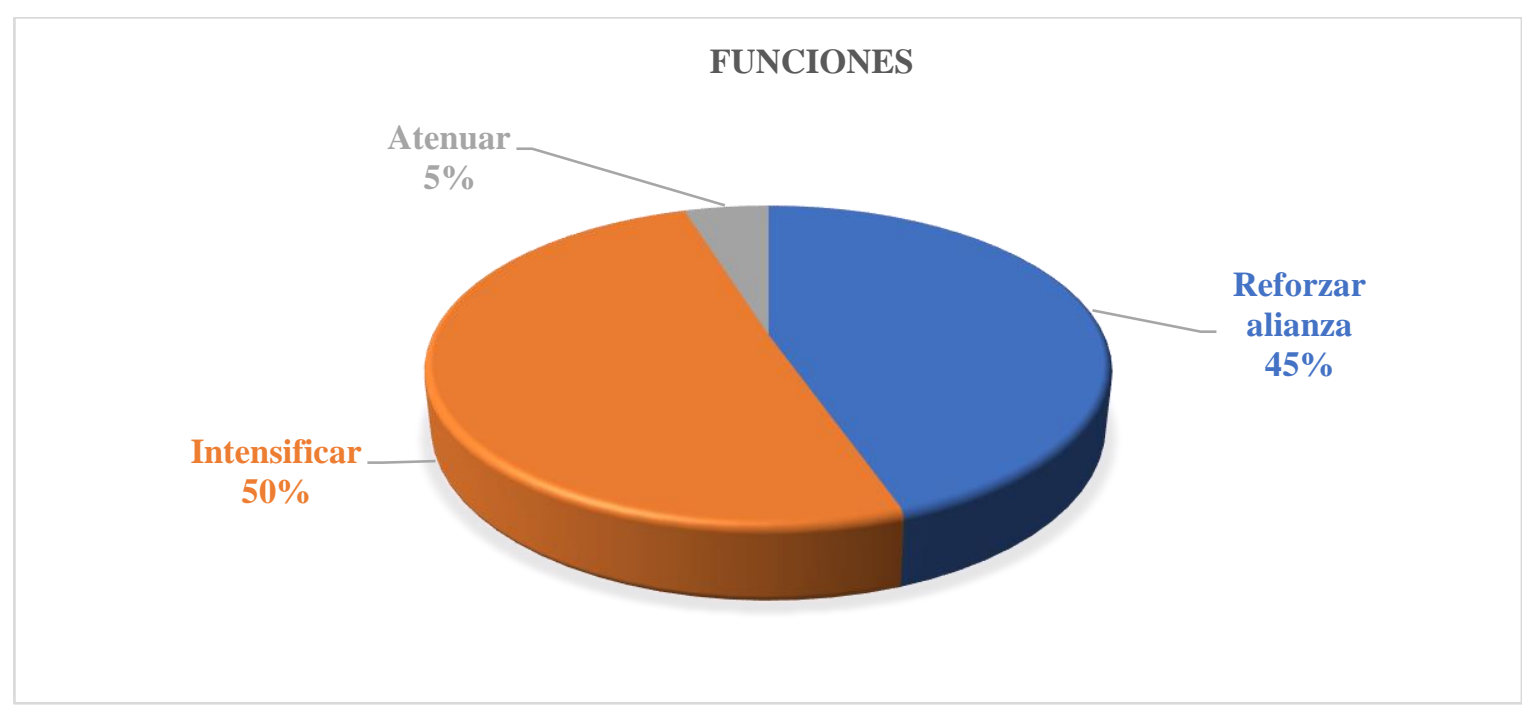

Tabla III: Proporciones de las funciones de ¿eh? en posición final

\section{2 ¿Eh? con valor atenuante}

A continuación analizaremos ejemplos que tienen lugar en entornos como el colegio, las casas de los hablantes, cafeterías, la calle, parques, etc. Existe la misma relación de poder entre los hablantes y los interlocutores comparten conocimientos comúnes. Los rasgos situacionales son (-) Formal. En el análisis de los ejemplos, se especifican los tipos de actos de habla, la implicación de las imágenes de los hablantes, los actos asertivos atenuados, en consonancia con lo expuesto por Albelda (2010) y Briz y Albelda (2013).

El marco físico del primer ejemplo analizado es el colegio; están en clase de manualidades en un entorno distendido, por lo que se deduce que el rasgo situacional es (-) Formal. Hablan sobre la gripe. Mateo le dice a Paco: no hue\% no hueles el peligro ¿eh? El acto de habla aseverativo no hue\% no hueles el peligro es ilocutivamente una crítica de Paco. Podría dañar su imagen, y, por ello, la atenuación de este acto de habla estaría justificada. Resulta, a nuestro juicio, más atenuado el enunciado "no hueles el peligro ¿eh?” que no "hueles el peligro". Al escuchar la grabación del sonido de esta conversación, se percibe que Mateo habla en un tono amigable en el que la entonación, al enunciar la partícula ¿eh?, es ascendente.

1. Mateo: tienes la gripe túl

Paco: $\quad$ quién me ha pegado la gripe/

Mateo: no hue\% no hueles el peligro ¿eh?

Paco: $\quad$ quién me ha pegado la gripe/

Mateo: $\quad$ y eso a qué viene/

Paco: $\quad$ qué por quién tengo gripe

MALCE2

Abundan los ejemplos de la partícula ¿eh? en enunciados claramente exhortativos entre los jóvenes de Madrid del corpus COLAm. Es el caso del ejemplo 2, en el que Cris y Paco, de 16-17 años, están hablando en un parque con otros amigos. Paco le dice a Cris: busca pero no te lo cojas todo ... ¿eh? Es un acto de habla directivo con fuerza ilocutiva exhortativa, que podría afectar la imagen de Cris y la imagen del mismo Paco, que podría parecer egoísta. Por ello mismo, este añade inmediatamente una justificación de la orden: "que es mio": 
2. Cris: ¿qué nos has comprado Paquito?

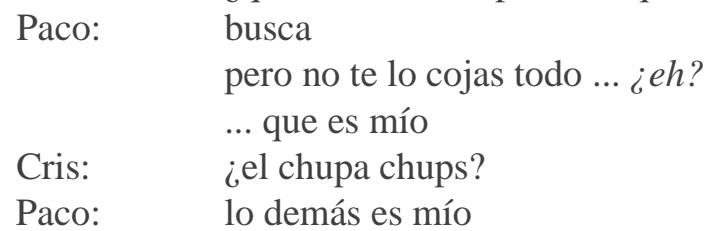

MABPE2

En el ejemplo 3 Marcos y Borja están con amigos en casa de uno de ellos. Están hablando de temas diferentes mientras fuman marihuana. Marcos le dice a Borja que no le apague su "rulo" de marihuana: a mí no me apagues ¿eh?:

3. Marcos: ¿tú has fumado fuera o no?

Borja: nunca quiere vacilar nunca quiere que le vaciles a él

Marcos: a mí no me apagues ¿eh?

Borja: ¿eh?

Marcos: $\quad$ no me apagues

Borja: $\quad$ Marcos Marcos

MAESB2

Considerando los factores del contexto mencionados por Albelda (2010a), se trata de un acto (-) formal, una orden cuyo posible daño a la a la imagen del oyente, Borja, explica que se atenúe con el recurso a la partícula ¿eh? En este mismo sentido, Fran, Mar y Luz están en casa de Mar en el caso 4. Mar y Luz le hacen la cera a Fran. Hay un tono jocoso y de confianza por la igualdad y la amistad que hay entre los participantes por la actividad que tienen entre manos. Mar le dirige el acto de habla directivo no grites ¿eh? a Fran. Es una orden atenuada, ya que podría amenazar la imagen de Fran:

4. Mar: eh venga.. a ver

no grites ¿eh?

Fran: $\quad$ no me vas a echar más

Luz: $\quad$ coño

Mar: no yo ya me voy $\quad$ MAMTE2

En el siguiente caso, ejemplo 5, Lucía emite un acto de habla recriminante que podría amenazar la imagen de Mar y de Loly: qué qué qué drogadictos sois ¿eh? Por ello, se atenúa la intervención mediante el ¿eh?:

5. José: $\quad$ a ahora nos pellizcamos

Loly: $\quad$ eh ah cabrón

me ha metido un pellizco en el culo sabes/

Mar: $\quad$ vamoosss

Lucía: qué qué qué drogadictos sois ¿¿eh?

Mar: míralo

Lucía: $\quad$ sois todos unos putos yonkis

MABPE2J

\section{3 ¿Eh? entre atenuar y solicitar ratificación a lo dicho}

El valor del apéndice interrogativo ¿eh? también tiene, en ocasiones, un valor entre atenuante y expresa duda al solicitar la ratificación del oyente. En la siguiente conversación, Trini, Luz y Ana están con otros amigos en una cafetería, Trini le dice a Ana que vaya a comprarle una funda de móvil, pero le dice que no puede ir ahora: ahora no puedes ir a 
comprarlo ¿eh? El acto de habla emitido por Trini es una advertencia que podría amenazar la imagen de Ana, a la vez que expresa duda:

6. Trini: ju ju ju si te digo la verdad no

Luz: $\quad$ pues si puedes

Trini: $\quad$ ahora no puedes ir a comprarlo ¿eh?

Luz: me lo compras o te lo dejo/

Ana: $\quad$ si de todos modos ahora no iba a comprarlo

(MABPE2)

En la banda sonora se escucha que Trini da la orden con entonación descendiente y no parece tener intención de atenuar, más bien parece dudar, lo que implicaría una petición de ratificación de la orden.

En ejemplo 7. es un acto de habla de una aseveración negativa: no se oye nada ¿eh? Los jóvenes revisan las grabadoras recibidas con las grabaciones hechas para el corpus COLAm. Mar y Manu están hablando de las grabaciones con unos amigos en un banco de la calle. Mar refuerza la advertencia/afirmación de no se oye nada con ¿eh? Este ¿eh? parece atenuar un acto de habla con una posible aseveración incómoda para la persona encargada de las grabaciones que se halla atenuada mediante el ¿eh? interrogativo final. Es exponente de cierta duda:

7. Manu: tú habla como si no estuviese el micrófono este

Mar: $\quad$ tronco pues sí

Manu: [qué es lo que nos han dicho/]

Mar: [no. no se oye nada ¿eh?]

MALCE2

Mar habla muy despacio, afirmando que no se oye nada en un tono relajado. En este caso también cabe pensar que el ¿eh? pide corroboración de la pregunta no se oye nada ¿eh? Si hubiera dicho no se oye nada ¿no? indicaría incertidumbre, cosa que con el uso de ¿eh? también tiene lugar. Con el ¿eh? interrogativo, Mar pide una corroboración ante una duda, no una respuesta explícita.

En el intercambio verbal 10., Luz, Mar y Fran están en casa de Luz. Mar y Fran están haciéndole la cera a Fran. La afirmación de Luz: no estaba seco ¿eh? es un acto de habla aseverativo, contrario a lo antes dicho y la presencia de la partícula ¿eh? atenúa esta aseveración. Puede amenazar la imagen de Mar, que había esperado lo contrario y dice: ¿a ver?

8. Fran: jim jim jim je je je je

Luz: [no estaba seco ¿eh?]

Fran: [como mola]

Mar: [a ver/]

Fran: [como mola hazme otro]

Luz: $\quad$ sí/

MAMTE2

Por lo tanto, ¿eh? podría atenuar la información negativa de no estaba seco, pero parece que Luz pide que refuercen la propia afirmación. Si se realizara la sustitución de ¿eh? por ¿no?: no estaba seco ¿no? la afirmación resulta menos fuerte que: no estaba seco. La grabación presenta una entonación de no estaba seco ¿ eh? de duda. 


\section{Observaciones finales}

\subsection{Limitaciones}

Para identificar el valor atenuante de ¿eh? en la interacción juvenil se han tenido que superar algunos obstáculos, entre ellos la escasez de intervenciones atenuadas por ¿eh? Este porcentaje tan bajo se debe a varios motivos: en el contexto informal del habla adolescente se tiende más a la hipérbole que a la atenuación. Debido a que forman grupos entre amigos cercanos, en las conversaciones coloquiales no (ab)usan de la atenuación. Los marcadores juveniles tienden a ser polifuncionales. Los estudios sobre la atenuación en relación con ¡eh? son límitados. Briz y Albelda afirman, además, que el valor atenuante no es siempre fácil de establecer, dado que puede tener varias funciones en un enunciado por su naturaleza polifuncional (2013). A esto se añade que por la naturaleza de la interacción adolescente no es fácil determinar los ejemplos de ¿eh? con valor atenuante porque los chicos frecuentemente bromean y en ocasiones, es difícil dar con la función exacta de los elementos de sus intervenciones. En consecuencia, hay casos que podrían ser tanto de atenuación como de pedir corroboración de lo que se ha afirmado.

En añadidura a aplicar los pasos propuestos por Albelda (2010a) y Briz y Albelda (2013) para delimiter el valor atenuante de ¿eh? también se ha usado el método de sustituir ¿eh? por un vacío u otra partícula atenuante (Blas Arroyo 1995). Ha sido eficaz escuchar los archivos de sonido para identificar los fines de los diferentes usos de ¿eh? Dados estos pasos, queda patente la existencia de un valor atenuante en la partícula discursiva ¿eh? en los casos analizados de lenguaje adolescente.

\subsection{Conclusiones}

La función principal de la partícula discursiva ¿eh?, cuyo significado viene moderado por la entonación (Hidalgo 2016), es mantener el contacto social. Esto le permite adquirir ciertas funciones de atenuación en algunos casos, añadida a la función principal de mantener la relación social.

La atenuación con ¿eh? final interrogativo aparece en 111 de un total de 2311 concordancias (4,8\% del total) del corpus COLAm, que contiene 500.000 palabras en total.

El adolescente hace uso de la partícua discursiva interrogativa ¿eh?, al final de los enunciados observados, con un valor de atenuante en la intervención de los actos de habla que implican una amenaza a la imagen del oyente. Hay casos dudosos de atenuación, que parecen pedir corroboración a una petición u orden, a la vez que atenúan, siempre de acuerdo con el fin social de su interacción.

Este es un tema que invita a ulteriores indagaciones.

\section{Bibliografía}

Albelda, Marta. 2005. "El refuerzo de la imagen social en conversaciones coloquiales en español peninsular." Estudios de la (des)cortesía en español: categorías conceptuales y aplicaciones a corpora orales y escritos, editado por Diana Bravo. Buenos Aires: Ediciones Dunken. 
Albelda, Marta. 2008. "Influencia de los factores situacionales en la codificación e interpretación de la descortesía." Pragmatics. Quarterly Publication of the International Pragmatics Association 18 (4):751-773.

Albelda, Marta. 2010a. ¿Cómo se reconoce la atenuación? Una aproximación metodológica basada en el español peninsular hablado." (Des)cortesía en español. Espacios teóricos y metodológicos para su estudio, editado por Franca Orletti y Laura Mariottini. Roma: Università Roma Tré y Programa EDICE.

Albelda, Marta. 2010b. "Estudio sociolingüístico (piloto) de la atenuación en el corpus PRESEEA de Valencia." Actas del IX Congreso Internacional de Lingüística General.

Albelda, Marta y Cestero Mancera, Ana María. 2011. "De nuevo, sobre los procedimientos de atenuación lingüística." Español Actual 96: 9-40.

Albelda, Marta y Briz, Antonio. 2013. "Una propuesta teórica y metodológica para el análisis de la atenuación lingüística en español y portugués." Onomázein 28: 288-319.

Blas Arroyo, José Luis. 1995. "La interjección como marcador discursivo: el caso de "eh"." Anuario de Lingüística Hispánica XI: 81-117.

Blas Arroyo, José Luis. 2005. Sociolingüística del español desarrollos y perspectivas en el estudio de la lengua española en contexto social, Lingüística. Madrid: Cátedra.

Briz, Antonio. 2003. "La interacción entre jóvenes: Español coloquial, argot y lenguaje juvenil." Lexicografía y Lexicología en Europa y América: Homenaje a Günther Hensch, editado por María Teresa Echenique Elizondo y Sánchez Méndez, Juan Pedro. Madrid: Gredos.

Briz, Antonio. 2006. "Atenuación y cortesía verbal en la conversación coloquial. Su tratamiento en la clase de ELE." Actas del programa de formación para profesorado de ELE 2005-2006, editado por Carmen Pastor Villalba. Múnich: Instituto Cervantes, 2007.

Briz, Antonio. 2007. "Atenuación y cortesía verbal en España y en América. Para un análisis semántico, pragmático y sociopragmático." En El español de América, Actas del VI Congreso Internacional, editado por César Hernández Alonso y Castañeda San Cirilo, Leticia. Valladolid: Diputación Provincial de Valladolid.

Briz, Antonio y Pons, Salvador. 2008. Diccionario de partículas discursivas del español. En línea: www.dpde.es editado por Antonio Briz.

Briz, Antonio. 2010. "Lo coloquial y lo formal, el eje de la variedad lingüística." De moneda nunca usada. Estudios dedicados a José M. ${ }^{a}$ Enguita Utrilla, editado por Institución Fernando el Católico. Zaragoza: Diputación de Zaragoza.

Briz, Antonio y Albelda, Marta. 2013. "Una propuesta teórica y metodológica para el análisis de la atenuación lingüística en español y portugués. La base de un proyecto común." Onomázein 28:288-319.

Brown, B. Bradford, y Larson, James. 2009. "Peer Relationships in Adolescence." Handbook of Adolescent Psychology. New York: John Wiley \& Sons, Inc.

Brown, Penelope, y Levinson, Stephen. 1987. Politeness: Some Universals in Language Usage Studies in Interactional Sociolinguistics. Cambridge: Cambridge University Press. 
Carter, Ronald, y Mc Carthy, Michael. 2006. Cambridge grammar of English: a comprehensive guide: spoken and written English grammar and usage. Cambridge: Cambridge University Press

Cestero, Ana María. 2003. "El funcionamiento de los recursos lingüísticos de llamada de atención al interlocutor en la conversación y en el discurso académico." Pragmalingüística 10-11: 51-94.

Cestero, Ana María, Albelda, Marta y Briz, Antonio. 2015. "Estudio coordinado de la «Atenuación» en el marco de PRESEEA: Propuesta metodológica." Contribuciones para os estudios linguisticos e filológicos, editado por Dermeval da Hora, Lopes Pedrosa, Juliene y M. de Lucena, Rubens. Paraiba: Ideia.

Eckert, Penelope. 2003. "Language And Adolescent Peer Groups." Journal of Language and Social Psychology 22: 112-118.

García Vizcaíno, María José. 2005. "El uso de los apéndices modalizadores ¿no? y ¿eh? en español peninsular." Selected proceedings of the Second Workshop on Spanish Sociolinguistics, 89-101.

Gille, Johan. 2006. "“Este hombre se debe de mover, vamos": un primer acercamiento a los apéndices conversacionales." Micro-pragmatique des langues romanes editado por Maj-Britt Mosegaard Hansen. Roskilde: Department of Language and Culture, Roskilde University.

Gina Tomé, Margarida, de Matos, Gaspar Simões, Celeste, Inês Camacho, y Alves Diniz, José. 2012. "How Can Peer Group Influence the Behavior of Adolescents: Explanatory Model." Global Journal of Health Science (4(2)):26-35. doi: 10.5539/gjhs.v4n2p26.

Herrero, Gemma. 2002. "Aspectos sintácticos del lenguaje juvenil." El lenguaje de los jóvenes, editado por Félix Rodríguez, 67-96. Madrid: Ariel Social.

Hidalgo Navarro, Antonio. 2016. El estudio de la entonación del español hablado: una visión retrospectiva en el umbral del s. XXI, editado por Ana $\mathrm{M}^{\mathrm{a}}$ Fernández Planas. Barcelona.

Jørgensen, Annette Maria Myre, y Martínez, Juan Antonio. 2007. "Los marcadores del discurso del lenguaje juvenil de Madrid”. ReVEL, 9 vol. 5.

Jørgensen, Annette Myre. 2009. "En plan used as a hedge in Spanish teenage language." Youngspeak in a Multilingual Perspective, editado por Anna Brita Stenström y Myre Jørgensen, Annette. Amsterdam: John Benjamins Publishing Company.

Jørgensen, Annette Myre. 2011. "Los vocativos en el lenguaje juvenil de Santiago de Chile y Madrid." Estudios de variación pragmática en español, editado por by Carmen García y Placencia, Maria Elena. Buenos Aires: Dunken.

Jørgensen, Annette Myre, y Drange, Eli-Marie. 2012. "La lengua juvenil de las metrópolis Madrid y Santiago de Chile." Arena Romanistica 9: 74-96.

Jørgensen, Annette Maria Myre. 2013. "Spanish teenage Language and the COLAm corpus." The many facets of corpus linguistics in Bergen. In honour of Knut Hofland editado por Lidun Hareide, Johansson, Christer y Oakes, Michael, BeLLS Vol 3, Nr. 1.

Jørgensen, Annette Maria Myre, y Eguía Padilla, Esperanza. 2015. "Presentación de COLA, un corpus oral de lenguaje adolescente en línea." Actes du XIXème Congrés des romanistes scandinaves. Reykjavik: Háskoli Islands. 
Malinowski, Bronislaw. 1999 [1926]. "On Phatic Communion." The Discourse Reader, editado por Adam Jaworski y Coupland, Nikolas, 302-305. London and New York: Routledge.

Montañez Mesas, Marta Pilar. 2007. "Marcadores del discurso y posición final: La forma ¿eh? en la conversación coloquial española." ELUA: 1-20.

Montañez Mesas, Marta Pilar. 2008. "El apéndice ¿No? en la conversación coloquial española." Boletín de Filología 2 (Tomo XLIII): 117-174.

Ortega Olivares, Jenaro. 1985. "Apéndices modalizadores en español: los comprobativos." Estudios románicos dedicados al profesor Andrés Soria Ortega: en el XXV aniversario de la Cátedra de Literaturas Románicas, 239-255. Granada: Servicio de Publicaciones de la Universidad de Granada.

Olivares Ortega, Jenaro. 1986. "Aproximación al mecanismo de la conversación." VERBA 13: 269-290.

Rodríguez, Félix. 2001. El lenguaje de los jóvenes. Madrid: Ariel Social.

Santos Ríos, Luis. 2003. Diccionario de partículas. Badajoz: Luso-española de ediciones.

Stenström, Anna-Brita. 2005. "It is very good eh - Está muy bien eh. Teenagers' use of tags London and Madrid compared." Contexts - Historical, Social, Linguistic. Studies in Celebration of Toril Swan, editado por Kevin Mc Cafferty, Bull, Tove y Killie, Kristin. Berlin: Elsevier.

Val.Es.Co, Grupo. 2014. "Las unidades del discurso oral. La propuesta Val.Es.Co. de segmentación de la conversación (coloquial)." Estudios de Lingüística del Español 35 (1): 11-71.

Vigara Tauste, Ana María. 1990. "La función fática en el lenguaje." Actas del Congreso de la Sociedad Española de lingüística. XX Aniversario (II): 9.

Zimmermann, Klaus. 2002. "La variedad juvenil y la interacción verbal entre jóvenes." El lenguaje de los jóvenes, editado por Félix Rodríguez. Barcelona: Ariel. 